\title{
Novel Robust Optimization and Power Allocation of Time Reversal-MIMO-UWB Systems in an Imperfect CSI
}

\author{
Sajjad Alizadeh and Hossein Khaleghi Bizaki
}

\begin{abstract}
Time Reversal (TR) technique is an attractive solution for a scenario where the transmission system employs low complexity receivers with multiple antennas at both transmitter and receiver sides. The TR technique can be combined with a high data rate MIMO-UWB system as TRMIMO-UWB system. In spite of TR's good performance in MIMO-UWB systems, it suffers from performance degradation in an imperfect Channel State Information (CSI) case. In this paper, at first a robust TR pre-filter is designed together with a MMSE equalizer in TR-MIMO-UWB system where is robust against channel imperfection conditions. We show that the robust pre-filter optimization technique, considerably improves the BER performance of TR-MIMO-UWB system in imperfect CSI, where temporal focusing of the TR technique is kept, especially for high SNR values. Then, in order to improve the system performance more than ever, a power loading scheme is developed by minimizing the average symbol error rate in an imperfect CSI. Numerical and simulation results are presented to confirm the performance advantage attained by the proposed robust optimization and power loading in an imperfect CSI scenario.
\end{abstract}

Keywords - MMSE equalizer, Time Reversal (TR) technique, MIMO channel, Ultra-Wideband (UWB) system.

\section{INTRODUCTION}

Ultra-wide band (UWB) systems have recently received much interest from both research community and industry. One of the main applications of UWB system is its ability of high data rate transmission in indoor environments, where remarkable temporal channel resolution and so, high complexity of the receiver structure, are inevitable [1]-[2]. However, due to the wide bandwidth property, UWB systems suffer from a very long delay spread of multipath channels, especially in indoor environments.

On the other hand, transmission over a Multiple Input Multiple Output (MIMO) channel has been shown as one of the important techniques in modern communications because of its high spectral efficiency [3]. In order to benefit a high data rate system with high spectral efficiency, the UWB system can be used in MIMO channels as MIMO-UWB system. Time Reversal (TR) technique can be used to reduce the long delay spread of the UWB channel. TR can mitigate not only the Inter Symbol Interference (ISI) but also the Multi Stream Interference (MSI) caused by transmitting several data streams, simultaneously [4]-[6]. The main

Manuscript received May 27, 2013.

Sajjad Alizadeh is with the Electrical and Electronic Engineering University Complex, Malek Ashtar University of Technology, Tehran, Iran, (e-mail: s.alizadeh@chmail.ir).

Hossein Khaleghi Bizaki is with the Electrical and Electronic Engineering University Complex, Malek Ashtar University of Technology, Tehran, Iran, (e-mail: bizaki@ee.iust.ac.ir).

doi: $10.11601 /$ ijates.v2i2.49 advantages of the TR technique are: temporal and spatial focusing [6]. In temporal focusing, the received signal is compressed in the time domain. Owing to this property, the ISI caused by the original multipath channel is greatly reduced. Also, in spatial focusing, the received signal is focused on the intended user at some specific position which is determined by the transmitter or user that uses the corresponding channel to pre-filter the intended data signal. In this paper, the TR technique is considered to overcome both MSI and ISI in MIMO spatial multiplexing as a low cost, low power and low complexity receiver solution.

In a MIMO-UWB system, we have $N_{T} \times N_{R}$ multipath channels between transmitter and receiver sides, where $N_{T}$ and $N_{R}$ denote the number of transmit and receive antennas, respectively. Obtaining the TR pre-coding waveform for the $m^{\text {th }}$ transmit antenna and $N_{R}$ receive antennas is straightforward [4]. First, a sounding pulse is sent through all the $N_{R}$ antennas from receiver to the transmitter. Second, the received signals at each transmitter antenna are then recorded, digitized, and time reversed. If the sounding pulse is sufficiently short, we can directly use the time-reversed version of received signals as the TR pre-coder. Otherwise, deconvolution effort is necessary to remove the pulse effect from the received sounding signal [4]. Practically, if we use the deconvolution efforts (e.g. CLEAN Algorithm [7]-[8]); some errors may be caused in the channel estimation. In spite of TR's good performance, it is very sensitive to erroneous Channel State Information (CSI). Therefore the improved algorithm should be considered in a TR-MIMOUWB communication system in imperfect CSI.

Most of the research on TR pre-coding assumes that perfect CSI is given at the transmitter side [5], [9]-[10]. Authors in [5] proposed an antenna selection scheme for TR-MIMO-UWB communication system in a perfect CSI case to reduce the number of transmit antenna. Also, a MMSE equalizer is used for TR-UWB system with perfect CSI in [9] to mitigate the residual ISI and increase transmission data rate. Some power loading schemes are used in [10] for TR-MISO-UWB system with perfect CSI. Unfortunately, these methods suffer from the effects of the imperfect CSI in the transmitter.

On the other hand, the imperfect CSI has been considered in some of the research on TR-MIMO-UWB system, especially in recent years [6], [11-14]. The effect of channel imperfection on the TR-MIMO-UWB system performance has been evaluated in [6] for low and high data rate transmissions. Authors of this literature have practically shown that the TR system is almost robust in imperfect CSI where the robustness can be obtained with performance degradation. In the mentioned reference when the noise level 
of channel estimation is noticeable, using the TR method cannot yield satisfactory performance. Unfortunately, an optimization scheme has not been provided in this literature to overcome the effects of the channel imperfection. In this paper, we propose a pre-processing method as robust optimization to compensate the estimation error in an imperfect CSI scenario for MIMO-UWB systems.

The robustness of TR technique in imperfect CSI caused by a time varying channel environment has been studied in [11] by experimental results. It has been shown that, if the channel maintains some partial correlation with the previous channel, the TR method can give a good performance even if the total correlation of the channels is very low. But if the correlation of the channels is not available, they have not proposed a theoretical solution for system performance improvement. Also, a post-time-reversed MIMO-UWB transmission scheme has been proposed in [12] which improves the TR robustness against imperfect CSI caused by channel estimation error when compared with the conventional TR scheme. We propose simpler robust optimization schemes, in this paper, with the same attained performance. Also, in our previous works [13]-[14], we had provided the pre-filtering solutions for the channel estimation error compensation by using the channel estimation error covariance matrix for the Single-Input Single-Output (SISO) Time Reversal UWB systems. In this paper, similar approaches (pre-filtering method) with a procedure different from these literatures are used to analysis and improve the TR-MIMO-UWB systems in an imperfect CSI scenario. Therefore, based on mentioned researches on TR pre-coding and also, works of [13]-[16], this paper proposes a novel pre-filter optimization and power allocation scheme at transmitter side to improve the TR-MIMO-UWB system performance in an imperfect CSI scenario.

The rest of the paper is organized as follow; we introduce the system model in Section II. In Section III, a novel robust optimization scheme is derived based on a MMSE equalizer in the TR-MIMO-UWB system. The power adaptation policies optimizing the average symbol error rate performance are derived for imperfect CSI in Section IV. Numerical and simulation results characterizing the performance of the proposed methods are presented in Section V, and finally, conclusions are drawn in Section VI.

\section{TR-MIMO-UWB SYSTEM MODEL}

The TR-MIMO-UWB system is depicted in Fig. 1 with $N_{T}$ transmit and $N_{R}$ receive antennas. Let us consider a UWB system using binary pulse amplitude (BPAM) modulation with pulse shaping according to FCC desired power spectrum density [1]-[2]. The input signal is converted into $N_{R}$ streams, pre-coded with TR pre-filter, and then sent to $N_{T}$ transmitting antennas, simultaneously. The resultant signal passes through the multipath MIMO channel and then, is corrupted by an AWGN. Thus, there are $N_{T} \times N_{R}$ multipath channel between transmit and receive antennas. For simplicity of analysis, we assume that the maximum length of each channel realization is $L$ [5]-[6]. The TR pre-filter is used in the spatial multiplexing UWB system in order to cope with the ISI and MSI problems. Finally, after passing the received signal through the MMSE equalizer, the data stream is detected based on a threshold value $v_{T}$.

The effect of channel estimation error can be considered as $\boldsymbol{H}=\hat{\boldsymbol{H}}+\boldsymbol{\Delta} \boldsymbol{H}$ where $\boldsymbol{H}, \hat{\boldsymbol{H}}$ and $\boldsymbol{\Delta H}$ are the true value, estimated value and estimation error of the channel impulse response (CIR), respectively. It is assumed that the entries of $\boldsymbol{\Delta H}$ are i.i.d. random variables with zero mean complex Gaussian noise. The pre-filter with respect to the estimation errors can be modeled as $\boldsymbol{H}_{T}=\hat{\boldsymbol{H}}_{T}+\boldsymbol{F}_{\boldsymbol{C}}$ where $\boldsymbol{H}_{T}, \hat{\boldsymbol{H}}_{T}$ and $\boldsymbol{F}_{\boldsymbol{C}}$ are the robust TR pre-filter, the conventional TR pre-filter and the compensator pre-filter, respectively. The estimated CIR between $\boldsymbol{i}$-th transmit antenna and $\boldsymbol{j}$-th receive antenna can be denoted as

$\hat{h}_{i j}(t)=\sum_{l=1}^{L} \hat{\alpha}_{l}^{i j} \delta\left(t-\hat{\tau}_{l}^{i j}\right) ; i=1, \ldots, N_{T} \quad, \quad j=1, \ldots, N_{R}$

where $\hat{\alpha}_{l}$ is the estimated amplitude, $\hat{\tau}_{l}$ is the delay of the $l$ th tap and $L$ is the maximum delay spread. The discrete time vector form of CIR in reversed order is denoted as: $\hat{\boldsymbol{h}}_{i j}=\left[\hat{h}_{i j}[L-1], \ldots, \hat{h}_{i j}[l], \ldots, \hat{h}_{i j}[0]\right]_{1 \times L}$. Also the estimation error vector of the CIR is defined as: $\Delta \boldsymbol{h}_{i j}=\left[\Delta h_{i j}[L-1], \ldots, \Delta h_{i j}[l], \ldots, \Delta h_{i j}[0]\right]_{1 \times L}$ where $i=1, \ldots, N_{T}$ $, j=1, . ., N_{R}$ and $l=0, . ., L-1$. In matrix form we have [5]

$$
\begin{gathered}
\hat{\boldsymbol{H}}=\left(\begin{array}{cccc}
\hat{\boldsymbol{h}}_{11} & \hat{\boldsymbol{h}}_{21} & \cdots & \hat{\boldsymbol{h}}_{N_{T} 1} \\
\hat{\boldsymbol{h}}_{12} & \hat{\boldsymbol{h}}_{22} & \cdots & \hat{\boldsymbol{h}}_{N_{T} 2} \\
\vdots & \vdots & & \vdots \\
\hat{\boldsymbol{h}}_{1 N_{R}} & \hat{\boldsymbol{h}}_{2 N_{R}} & \cdots & \hat{\boldsymbol{h}}_{N_{T} N_{R}}
\end{array}\right)_{N_{R} \times N_{T} L} \\
\boldsymbol{\Delta H}=\left(\begin{array}{cccc}
\boldsymbol{\Delta} \boldsymbol{h}_{11} & \boldsymbol{\Delta} \boldsymbol{h}_{21} & \cdots & \boldsymbol{\Delta} \boldsymbol{h}_{N_{T} 1} \\
\boldsymbol{\Delta} \boldsymbol{h}_{12} & \boldsymbol{\Delta \boldsymbol { h } _ { 2 2 }} & \cdots & \boldsymbol{\Delta} \boldsymbol{h}_{N_{T} 2} \\
\vdots & \vdots & & \vdots \\
\boldsymbol{\Delta} \boldsymbol{h}_{1 N_{R}} & \boldsymbol{\Delta \boldsymbol { h } _ { 2 N _ { R } }} & \cdots & \boldsymbol{\Delta} \boldsymbol{h}_{N_{T} N_{R}}
\end{array}\right)_{N_{R} \times N_{T} L}
\end{gathered}
$$

If estimated CIR of all channels $\hat{\boldsymbol{h}}_{i j}$ are known at the transmitter side, time reversed version of them are used to pre-filter the transmit data. Thus we can construct the prefilter matrix based on the time reversed form of the estimated channels which is an $N_{T} L \times N_{R}(2 L-1)$ matrix as [6]

$$
\hat{\boldsymbol{H}}_{T}=\left(\begin{array}{cccc}
\overline{\boldsymbol{H}}_{11} & \overline{\boldsymbol{H}}_{12} & \ldots & \overline{\boldsymbol{H}}_{1 N_{R}} \\
\overline{\boldsymbol{H}}_{21} & \overline{\boldsymbol{H}}_{22} & \ldots & \overline{\boldsymbol{H}}_{2 N_{R}} \\
\vdots & \vdots & & \vdots \\
\overline{\boldsymbol{H}}_{N_{T} 1} & \overline{\boldsymbol{H}}_{N_{T} 2} & \cdots & \overline{\boldsymbol{H}}_{N_{T} N_{R}}
\end{array}\right)_{N_{T} L \times N_{R}(2 L-1)}
$$

where each sub-matrix $\overline{\boldsymbol{H}}_{i j}$ is an $L \times(2 L-1)$ Toeplitz matrix defined by [6] as

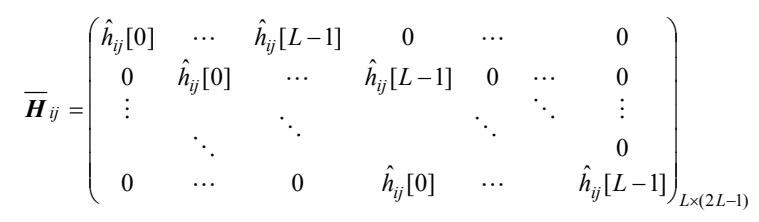




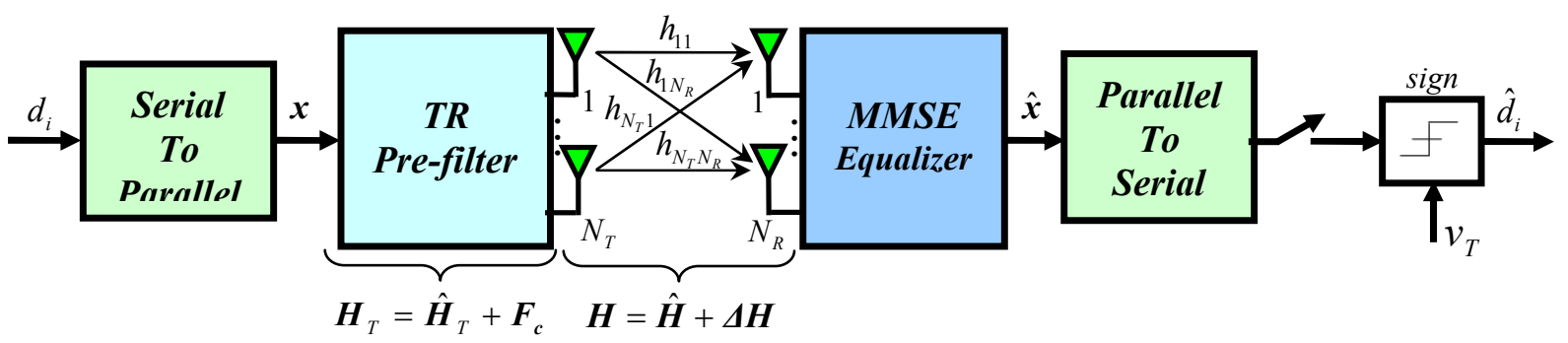

If we define

$$
\boldsymbol{F}_{c}=\left(\begin{array}{cccc}
\overline{\boldsymbol{F}}_{11} & \overline{\boldsymbol{F}}_{12} & \cdots & \overline{\boldsymbol{F}}_{1 N_{R}} \\
\overline{\boldsymbol{F}}_{21} & \overline{\boldsymbol{F}}_{22} & \cdots & \overline{\boldsymbol{F}}_{2 N_{R}} \\
\vdots & \vdots & & \vdots \\
\overline{\boldsymbol{F}}_{N_{T} 1} & \overline{\boldsymbol{F}}_{N_{T} 2} & \cdots & \overline{\boldsymbol{F}}_{N_{T} N_{R}}
\end{array}\right)_{N_{T} L \times N_{R}(2 L-1)}
$$

then each sub-matrix $\overline{\boldsymbol{F}}_{i j}$ is an $L \times(2 L-1)$ Toeplitz matrix defined by

$$
\overline{\boldsymbol{F}}_{i j}=\left(\begin{array}{ccccccc}
f_{i j}[0] & \ldots & f_{i j}[L-1] & 0 & \ldots & & 0 \\
0 & f_{i j}[0] & \cdots & f_{i j}[L-1] & 0 & \ldots & 0 \\
\vdots & \ddots & \ddots & & \ddots & \ddots & \vdots \\
0 & \cdots & 0 & f_{i j}[0] & \cdots & & f_{i j}[L-1]
\end{array}\right)_{L \times(2 L-1)}
$$

The goal is the calculation of the error compensator matrix $\boldsymbol{F}_{c}$ with the assumption that the probability distribution of the error coefficients are as: $\Delta h_{i j}[l] \sim C N\left(0, \sigma_{e}^{2}\right)$. Also, $\quad \boldsymbol{C}_{\boldsymbol{\Delta H}}=E\left\{\boldsymbol{\Delta} \boldsymbol{H}^{H} \boldsymbol{\Delta} \boldsymbol{H}\right\} \quad$ and $E\{\boldsymbol{\Delta H}\}=0$ are assumed to be known.

The received symbols vector at the MIMO channel output with TR pre-filter can be written as $\boldsymbol{y}=\boldsymbol{H}_{T R} \boldsymbol{x}+\boldsymbol{n}$ where $\boldsymbol{x}=\frac{1}{\sqrt{N_{T}}}\left[x_{1}, x_{2}, \ldots, x_{N_{T}}\right]^{T}, \quad \boldsymbol{y}=\frac{1}{\sqrt{N_{T}}}\left[y_{1}, y_{2}, \ldots, y_{N_{R}}\right]^{T}$, $\boldsymbol{n}=\left[n_{1}, n_{2}, \ldots, n_{N_{R}}\right]^{T}$ and $\boldsymbol{H}_{T R}=\boldsymbol{H}_{T}$ are the transmitted symbols, the received symbols, AWGN noise and equivalent TR response, respectively. The vector $\boldsymbol{x}$ contains i.i.d. random variables with zero mean $E\{\boldsymbol{x}\}=0$ and variance $E\left\{\boldsymbol{x} \boldsymbol{x}^{H}\right\}=\sigma_{\boldsymbol{x}}^{2} \boldsymbol{I}$. For simplicity of analysis, we assume $\boldsymbol{n} \sim C N\left(0, \sigma_{n}^{2} \boldsymbol{I}\right), \sigma_{\boldsymbol{x}}^{2}=1$ and also, the antipodal modulation is considered.

\section{ROBUST TR OPTIMIZATION BASED ON MMSE CRITERION}

It is desired to calculate the error compensator pre-filter $\boldsymbol{F}_{\boldsymbol{c}}$ in an imperfect CSI with the assumption that the matrices $\hat{\boldsymbol{H}}, \hat{\boldsymbol{H}}_{T}$ and $\boldsymbol{C}_{\boldsymbol{\Delta H}}$ are known. The error vector is considered as the difference between the transmitted symbols and the detected symbols, as $\boldsymbol{e}=\hat{\boldsymbol{x}}-\boldsymbol{x}$. Thus MMSE solution should minimize the cost function $E\left\{|\boldsymbol{e}|^{2}\right\}=E\left\{|\hat{\boldsymbol{x}}-\boldsymbol{x}|^{2}\right\}$ with respect to pre-filter $\boldsymbol{F}_{\boldsymbol{c}}$. For simplicity of analysis we use the orthogonality principle, instead of the MMSE solution [15]. In this case, the orthogonality principle can be considered as $E\left\{\boldsymbol{e} \boldsymbol{x}^{H}\right\}=0$ in which we obtain $E\left\{\hat{\boldsymbol{x}} \boldsymbol{x}^{H}\right\}=E\left\{\boldsymbol{x} \boldsymbol{x}^{H}\right\}$.
By considering the MMSE equalizer in the receiver side, the estimated symbol $\hat{\boldsymbol{x}}$ can be expressed as [9]

$\hat{\boldsymbol{x}}=\left(\boldsymbol{H}_{T R}^{H} \boldsymbol{H}_{T R}+\frac{1}{\rho} \boldsymbol{I}\right)^{-1} \boldsymbol{H}_{T R}^{H} \boldsymbol{y}$

where $\rho=\frac{\sigma_{x}^{2}}{\sigma_{n}^{2}}$ is the signal to noise ratio. By using (2), the cross-correlation between the estimated and the transmitted symbols can be calculated as

$E\left\{\hat{\boldsymbol{x}} \boldsymbol{x}^{H}\right\}=\sigma_{\boldsymbol{x}}^{2} E\left\{\left(\rho \boldsymbol{H}_{T R}^{H} \boldsymbol{H}_{T R}+\boldsymbol{I}\right)^{-1}\left(\rho \boldsymbol{H}_{T R}^{H} \boldsymbol{H}_{T R}\right)\right\}$

Because $\rho \boldsymbol{H}_{T R}^{H} \boldsymbol{H}_{T R}$ is symmetric, we can use the following approximation [17]

$\left(\rho \boldsymbol{H}_{T R}^{H} \boldsymbol{H}_{T R}\right)\left(\rho \boldsymbol{H}_{T R}^{H} \boldsymbol{H}_{T R}+\boldsymbol{I}\right)^{-1} \cong \frac{1}{\rho^{2}}\left(\boldsymbol{H}_{T R}^{H} \boldsymbol{H}_{T R}\right)^{-2}-\frac{1}{\rho}\left(\boldsymbol{H}_{T R}^{H} \boldsymbol{H}_{T R}\right)^{-1}+\boldsymbol{I}$

Thus, by using the above approximation, (3) can be written as

$E\left\{\hat{\boldsymbol{x}} \boldsymbol{x}^{H}\right\}=\frac{\sigma_{\boldsymbol{x}}^{2}}{\rho^{2}} E\left\{\left(\boldsymbol{H}_{T R}^{H} \boldsymbol{H}_{T R}\right)^{-2}\right\}-\frac{\sigma_{\boldsymbol{x}}^{2}}{\rho} E\left\{\left(\boldsymbol{H}_{T R}^{H} \boldsymbol{H}_{T R}\right)^{-1}\right\}+\sigma_{\boldsymbol{x}}^{2} \boldsymbol{I}$

As a result, based on the orthogonality principle, i.e., $E\left\{\hat{\boldsymbol{x}} \boldsymbol{x}^{H}\right\}=E\left\{\boldsymbol{x} \boldsymbol{x}^{H}\right\}$ we obtain

$E\left\{\boldsymbol{H}_{T R}^{H} \boldsymbol{H}_{T R}\right\}=\frac{1}{\rho} \boldsymbol{I}$

where $\boldsymbol{H}_{T R}=\hat{\boldsymbol{H}} \hat{\boldsymbol{H}}_{T}+\hat{\boldsymbol{H}} \boldsymbol{F}_{\boldsymbol{c}}+\boldsymbol{\Delta} \boldsymbol{H} \hat{\boldsymbol{H}}_{T}+\boldsymbol{\Delta} \boldsymbol{H} \boldsymbol{F}_{\boldsymbol{c}}$ is the equivalent TR channel response matrix of size $N_{R} \times N_{R}(2 L-1)$ which is given by

$$
\boldsymbol{H}_{T R}=\left(\begin{array}{cccc}
\overline{\boldsymbol{h}}_{11} & \overline{\boldsymbol{h}}_{21} & \cdots & \overline{\boldsymbol{h}}_{N_{R} 1} \\
\overline{\boldsymbol{h}}_{12} & \overline{\boldsymbol{h}}_{22} & \cdots & \overline{\boldsymbol{h}}_{N_{R} 2} \\
\vdots & \vdots & & \vdots \\
\overline{\boldsymbol{h}}_{1 N_{R}} & \overline{\boldsymbol{h}}_{2 N_{R}} & \cdots & \overline{\boldsymbol{h}}_{N_{R} N_{R}}
\end{array}\right)_{N_{R} \times N_{R}(2 L-1)}
$$

where each vector $\overline{\boldsymbol{h}}_{i j}$ is a $1 \times(2 L-1)$ autocorrelation $(i=j)$ or cross-correlation $(i \neq j)$ vector. Note that, the peak value of auto-correlation is located at the $L^{\text {th }}$ index. By TR method, the desired auto-correlation part in the equivalent CIR for the TR-MIMO scenario forms a strong peak and dominates in the $L^{\text {th }}$ sample of the received signal captured by any antenna [6].

We assume $\boldsymbol{\Delta} \boldsymbol{H}$ and $\hat{\boldsymbol{H}}$ are mutually independent. Substituting $\boldsymbol{H}_{T R}$ into (6), we have

$\hat{\boldsymbol{H}}_{T}^{H} \hat{\boldsymbol{H}}^{H} \hat{\boldsymbol{H}} \hat{\boldsymbol{H}}_{T}+\hat{\boldsymbol{H}}_{T}^{H} \hat{\boldsymbol{H}}^{H} \hat{\boldsymbol{H}} \boldsymbol{F}_{\boldsymbol{C}}+\boldsymbol{F}_{\boldsymbol{C}}^{H} \hat{\boldsymbol{H}}^{H} \hat{\boldsymbol{H}} \hat{\boldsymbol{H}}_{T}+\boldsymbol{F}_{\boldsymbol{C}}^{H} \hat{\boldsymbol{H}}^{H} \hat{\boldsymbol{H}} \boldsymbol{F}_{\boldsymbol{C}}$
$+\hat{\boldsymbol{H}}_{T}^{H} \boldsymbol{C}_{\boldsymbol{\Delta H}} \hat{\boldsymbol{H}}_{T}+\hat{\boldsymbol{H}}_{T}^{H} \boldsymbol{C}_{\boldsymbol{\Delta} \boldsymbol{H}} \boldsymbol{F}_{\boldsymbol{C}}+\boldsymbol{F}_{\boldsymbol{C}}^{H} \boldsymbol{C}_{\boldsymbol{\Delta H}} \hat{\boldsymbol{H}}_{T}+\boldsymbol{F}_{\boldsymbol{C}}^{H} \boldsymbol{C}_{\boldsymbol{\Delta} \boldsymbol{H}} \boldsymbol{F}_{\boldsymbol{C}}=\frac{1}{\rho} \boldsymbol{I}$

With some manipulating of (7) we have

$$
\left(\hat{\boldsymbol{H}}_{T}+\boldsymbol{F}_{\boldsymbol{C}}\right)\left(\hat{\boldsymbol{H}}_{T}+\boldsymbol{F}_{\boldsymbol{C}}\right)^{H}=\frac{1}{\rho}\left(\hat{\boldsymbol{H}}^{H} \hat{\boldsymbol{H}}+\boldsymbol{C}_{\boldsymbol{\Delta H}}\right)^{-1}
$$


Finally, the elements of the estimation error compensator matrix $\boldsymbol{F}_{\boldsymbol{C}}$ can be obtained from compensated pre-filter matrix $\boldsymbol{H}_{T}=\hat{\boldsymbol{H}}_{T}+\boldsymbol{F}_{\boldsymbol{C}}$ as

$$
\boldsymbol{H}_{T} \boldsymbol{H}_{T}^{H}=\frac{1}{\rho}\left(\hat{\boldsymbol{H}}^{H} \hat{\boldsymbol{H}}+\boldsymbol{C}_{\Delta \boldsymbol{H}}\right)^{-1}
$$

where the non-square complex-valued matrix $\boldsymbol{H}_{T}$ can be found through the singular value decomposition (SVD) of (9) as follows [18].

Given the $N_{T} L \times N_{R}(2 L-1)$ matrix $\boldsymbol{H}_{T}$, let $\boldsymbol{U}$ be the $N_{T} L \times$ $N_{T} L$ matrix whose columns are the orthogonal eigenvectors of $\boldsymbol{H}_{T} \boldsymbol{H}_{T}^{H}$, and $\boldsymbol{V}$ be the $N_{R}(2 L-1) \times N_{R}(2 L-1)$ matrix whose columns are the orthogonal eigenvectors of $\boldsymbol{H}_{T}^{H} \boldsymbol{H}_{T}$. Also, let $r$ be the rank of the matrix $\boldsymbol{H}_{T}$. Then, there is a SVD of $\boldsymbol{H}_{T}$ as $\boldsymbol{H}_{T}=\boldsymbol{U} \sum \boldsymbol{V}^{H}$ where the eigenvalues $\lambda_{1}, \ldots, \lambda_{r}$ of $\boldsymbol{H}_{T} \boldsymbol{H}_{T}^{H}$ are the same as the eigenvalues of $\boldsymbol{H}_{T}^{H} \boldsymbol{H}_{T}$ and also, for $1 \leq i \leq r$, let $\sigma_{i}=\sqrt{\lambda_{i}}$, with $\lambda_{i} \geq \lambda_{i+1}$. Then the $N_{T} L \times N_{R}(2 L-1)$ matrix $\sum$ is composed by setting $\sum_{i i}=\sigma_{i}$ for $1 \leq i \leq r$, and zero otherwise. Thus based on (9) we have $\boldsymbol{H}_{T} \boldsymbol{H}_{T}^{H}=\boldsymbol{U} \sum \boldsymbol{V}^{H} \boldsymbol{V} \sum^{H} \boldsymbol{U}^{H}=\boldsymbol{U} \sum \sum^{H} \boldsymbol{U}^{H}$, that is, the left-hand side is a square symmetric matrix, and the right-hand side represents its symmetric diagonal decomposition. The values $\sigma_{i}$ are referred to as the singular values of $\boldsymbol{H}_{T}$. Then for (9) we have $\boldsymbol{U} \sum \sum^{H} \boldsymbol{U}^{H}=\frac{1}{\rho}\left(\hat{\boldsymbol{H}}^{H} \hat{\boldsymbol{H}}+\boldsymbol{C}_{\boldsymbol{\Delta H}}\right)^{-1}$. As a result, the above discussion in the calculation of $\boldsymbol{H}_{T}$ from (9) can be expressed as the following subroutine in which a pseudocode is presented for the numerical implementation of the proposed robust TR optimization algorithm based on MMSE criterion. This code is expressed as

- Compute the SVD of matrix $\frac{1}{\rho}\left(\hat{\boldsymbol{H}}^{H} \hat{\boldsymbol{H}}+\boldsymbol{C}_{\boldsymbol{\Delta H}}\right)^{-1}$ in (9) and order its singular values, i.e., $\boldsymbol{U}_{1}, \Sigma_{1}$ and $\boldsymbol{V}_{\boldsymbol{1}}$ as $\left[\boldsymbol{U}_{\boldsymbol{1}}, \Sigma_{\boldsymbol{1}}, \boldsymbol{V}_{\boldsymbol{1}}\right]=\operatorname{svd}\left\{\frac{1}{\rho}\left(\hat{\boldsymbol{H}}^{H} \hat{\boldsymbol{H}}+\boldsymbol{C}_{\boldsymbol{\Delta H}}\right)^{-1}\right\}$.

- $\operatorname{Set} \boldsymbol{U}=\boldsymbol{U}_{\boldsymbol{1}}$.

- Construct a zero matrix as $\stackrel{\Delta}{\boldsymbol{O}}=\operatorname{zeros}\left(N_{T} L, N_{R}(2 L-1)-N_{T} L\right)$.

- Set $\sum=\left[\operatorname{sqrt}\left(\sum_{1}\right)\right.$ O $]$.

- Compute the SVD of matrix $\hat{\boldsymbol{H}}_{T}$ and order its singular values, i.e., $\boldsymbol{U}_{\boldsymbol{0}}, \quad \sum_{\boldsymbol{0}}$ and $\boldsymbol{V}_{\boldsymbol{0}}$ as: $\left[\boldsymbol{U}_{\boldsymbol{0}}, \sum_{\boldsymbol{0}}, \boldsymbol{V}_{\boldsymbol{0}}\right]=\operatorname{svd}\left\{\hat{\boldsymbol{H}}_{T}\right\}$.

- Set $\boldsymbol{H}_{T} \approx \boldsymbol{U} \sum \boldsymbol{V}_{\boldsymbol{0}}^{H}$.

\section{POWER LOADING}

\section{A. In Conventional TR-MIMO-UWB System}

In transmission over parallel channels, it can happen that we are faced with sub-channels that would require enormous transmit power to achieve acceptable bit or symbol error rates, especially in an imperfect CSI case. In this case it is beneficial, given the fixed amount of transmit power available, not to aim for equal error rates in all sub-channels, but perform an optimum power loading by minimizing the average bit error rate [16]. Some power allocation schemes are proposed in [10] to reduce the delay spread of the channel impulse response in the TR-MISO-UWB systems. Authors in [10] did not consider imperfect CSI case and also ISI and MSI effects on system performance in which their analysis is true in ideal and fantastic case. Therefore, based on previous findings such as [15] and [16], we propose a new power loading scheme for TR-MIMO-UWB systems by minimizing the average bit error rate (BER) at the receiver in an imperfect CSI scenario.

The received signal at the MIMO channel output with TR pre-filtering, as mentioned in Section 2, without the channel estimation error compensation is given by

$\boldsymbol{y}=(\hat{\boldsymbol{H}}+\boldsymbol{\Delta H}) \hat{\boldsymbol{H}}_{T} \boldsymbol{x}+\boldsymbol{n}$

where $\boldsymbol{\Delta} \boldsymbol{H}$ and $\boldsymbol{n}$ are assumed mutually independent and uncorrelated matrices. The received signal at $j^{\text {th }}$ receive antenna can be expressed by

$$
\begin{aligned}
y_{j}= & \underbrace{\sum_{i=1}^{N_{T}} \hat{\boldsymbol{h}}_{i j} \overline{\boldsymbol{H}}_{i j} x_{j}}_{\text {Signal }} \\
& +\underbrace{\sum_{i=1}^{N_{T}} \sum_{\substack{k=1 \\
k \neq j}}^{N_{R}} \hat{\boldsymbol{h}}_{i j} \overline{\boldsymbol{H}}_{i k} x_{k}}_{v_{j}}+\underbrace{\sum_{i=1}^{N_{T}} \sum_{k=1}^{N_{R}} \boldsymbol{\Delta} \boldsymbol{h}_{i j} \overline{\boldsymbol{H}}_{i k} x_{k}}_{\text {Interference }}+\underbrace{n_{j}}_{w_{j}}
\end{aligned}
$$

The first part of the received signal is the desired data symbol. In this part, the equivalent channel is the autocorrelation of channels. Also, $v_{j}$ and $w_{j}$ are the interference from other symbols and the interference from the channel estimation error, respectively. In $v_{j}$ and $w_{j}$ parts, the equivalent channel is the cross-correlation of channels, which is small generally in comparison with the former. Also, $v_{j}$ and $w_{j}$ terms in (11) appears as interference which degrades the performance of TR-MIMOUWB system, especially in imperfect CSI. Some of this interference can be reduced by a MMSE equalizer, but, we try to maximize the Signal to Interference plus Noise Ratio (SINR) more than ever by a power allocation scheme. The SINR at the $j^{\text {th }}$ received antenna is given by

$$
\operatorname{SINR}_{j}=\frac{\left\|\sum_{i=1}^{N_{T}} \hat{\boldsymbol{h}}_{i j} \overline{\boldsymbol{H}}_{i j}\right\|_{F}^{2}}{\left\|\sum_{i=1}^{N_{T}} \sum_{\substack{k=1 \\ k \neq j}}^{N_{R}} \hat{\boldsymbol{h}}_{i j} \overline{\boldsymbol{H}}_{i k}\right\|_{F}^{2}+E\left\{\left\|\sum_{i=1}^{N_{T}} \sum_{k=1}^{N_{R}} \boldsymbol{\Delta} \boldsymbol{h}_{i j} \overline{\boldsymbol{H}}_{i k}\right\|_{F}^{2}\right\}+E\left\{\left\|n_{j}\right\|_{F}^{2}\right\}}
$$

where $\|\bullet\|_{F}$ denotes the Frobenius norm. If we define $\boldsymbol{R}_{i j} \stackrel{\Delta}{=} \hat{\boldsymbol{h}}_{i j} \overline{\boldsymbol{H}}_{i j}$ as auto-correlation vector of the channel vector $\hat{\boldsymbol{h}}_{i j}$ and, $\boldsymbol{C}_{i k} \stackrel{\Delta}{\underset{k \neq j}{=}} \hat{\boldsymbol{h}}_{i j} \overline{\boldsymbol{H}}_{i k}$ as cross-correlation vector of $\hat{\boldsymbol{h}}_{i j}$ with other sub-channels then, the SINR at the $j^{\text {th }}$ received antenna is expressed as 


$$
\operatorname{SINR}_{j}=\frac{\sum_{i=1}^{N_{T}} \sum_{l=0}^{2 L-2}\left|\boldsymbol{R}_{i j}(l)\right|^{2}}{\sum_{i=1}^{N_{T}} \sum_{\substack{k=1 \\ k \neq j}}^{N_{R}} \sum_{l=0}^{2 L-2}\left|\boldsymbol{C}_{i k}(l)\right|^{2}+L \sigma_{e}^{2} \sum_{i=1}^{N_{T}} \sum_{k=1}^{N_{R}} \sum_{l=0}^{L-1}\left|\hat{\boldsymbol{h}}_{i k}(l)\right|^{2}+\sigma_{n}^{2}}
$$

where the estimation errors of the different sub-channels are assumed to be mutually independent, i.e., $\boldsymbol{\Delta} \boldsymbol{h}_{i j} \amalg \boldsymbol{\Delta} \boldsymbol{h}_{m n}$ where $\amalg$ is the symbol, standing for mutual independence. If the channel estimation is perfect and also, because of focusing property of TR, we can neglect the interference part. Then the SNR is

$$
S N R_{j}=\frac{\sum_{i=1}^{N_{T}} \sum_{l=0}^{2 L-2}\left|\boldsymbol{R}_{i j}(l)\right|^{2}}{\sigma_{n}^{2}}
$$

Performance of the TR-MIMO-UWB system depends on transceiver structure and the received signal properties, e.g., its probability density function (PDF). For $L t_{\mathrm{s}} \geq 5 n \mathrm{sec}$ where $t_{\mathrm{s}}$ is time resolution of the channel, the average numbers of paths is high, so using central limit theorem, the sum of a large number of independent, zeromean random variables form a Gaussian PDF for the path gain [19]-[20]. But for $L t_{\mathrm{s}}<5 n \mathrm{sec}$ path gain PDF isn't Gaussian and as the $L t_{\mathrm{s}}$ increases, the non-Gaussian shape tends more to Gaussian, and the densities become more bell shaped [20]. According to the above discussion, the average BER of the TR-MIMO-UWB system, assuming BPAM modulation, can be derived approximately from Eq. (13) as

$$
B E R \approx \frac{1}{N_{T}} \sum_{j=1}^{N_{T}} Q\left(\sqrt{2 \operatorname{SINR}_{j} p_{j}}\right)
$$

where $p_{j}, P_{T}=\sum_{j=1}^{N_{T}} p_{j}$ and $Q(x)$ are the transmit power assigned to $j^{\text {th }}$ transmit antenna, the total transmitted available power and the Marcum Q-function, respectively. $Q(x)$ is defined as

$Q(x)=\frac{1}{\sqrt{2 \pi}} \int_{x}^{+\infty} \exp \left(-t^{2} / 2\right) d t$

For simplicity, we assume $\sigma_{x}^{2}=1$, so that $P_{T}=N_{T}$. The optimum power allocation vector $\boldsymbol{P}=\left[p_{1}, \ldots, p_{N_{T}}\right]^{T}$ that minimizes the average BER, when an imperfect CSI is presented, can be obtained by introducing the Lagrange function as

$L_{G}=\frac{1}{N_{T}} \sum_{j=1}^{N_{T}} Q\left(\sqrt{2 S I N R_{j} p_{j}}\right)-\lambda\left(N_{T}-\sum_{j=1}^{N_{T}} p_{j}\right)$

With partial derivative we obtain

$\frac{\partial L_{G}}{\partial p_{j}}=\frac{-1}{2 N_{T} \sqrt{\pi}} \sqrt{\frac{\operatorname{SINR}_{j}}{p_{j}}} \exp \left(-\operatorname{SINR}_{j} p_{j}\right)+\lambda$

Solving $\partial L_{G} / \partial p_{j}=0$ for $p_{j}$, we can allocate the power to each transmit antenna in a manner that

$\left(\frac{1}{\operatorname{SINR}_{j}}\right) p_{j} \exp \left(\operatorname{SINR}_{j} p_{j}\right)=\frac{1}{4 N_{T}^{2} \lambda^{2} \pi}$

and finally
$p_{j}(\lambda)=\frac{1}{\operatorname{SINR}_{j}} W\left(\left(\operatorname{SINR}_{j}\right)^{2} \frac{1}{4 N_{T}^{2} \lambda^{2} \pi}\right)$

where $W(x)$ is the real valued Lambert's W-function defined as the inverse of the function $f(x)=x \exp (x)$ for $x \geq 0$, i.e., $W(x)=a \Leftrightarrow a \exp (a)=x \quad$ [21]. Since the $W(x)$ function is real and concave, the unique solution for power allocation vector can be found by the following simple iterative procedure [22]

- Choose a positive $\lambda$, which fulfils

$$
\sum_{j=1}^{N_{T}}\left(\operatorname{SINR}_{j}\right) \frac{1}{4 N_{T}^{2} \lambda^{2} \pi} \leq P_{T}
$$

- Calculate

$$
\hat{P}_{T}=\sum_{j=1}^{N_{T}} \frac{1}{\operatorname{SINR}_{j}} W\left(\left(\operatorname{SINR}_{j}\right)^{2} \frac{1}{4 N_{T}^{2} \lambda^{2} \pi}\right)
$$

- If $\hat{P}_{T}$ is not yet sufficiently close to $P_{T}$ then multiply $\lambda$ by $P_{T} / \hat{P}_{T}$ and go back to step (2).

- Compute $\boldsymbol{P}=\left[p_{1}, \ldots, p_{N_{T}}\right]^{T}$ according to (20).

Note that since $W(x)$ for $x>-1 / e$ is a monotonic function then according to $(20)$ the highest powers $\left(\max p_{j}\right)$ are assigned to the weakest signals so the SINR values approximately stay constant for all sub-channels.

\section{B. In Robust TR-MIMO-UWB System}

Proposed power loading scheme presented in Section 4.1 can be combined with robust optimization strategy mentioned in Section 3, but TR pre-filter should be calculated in an imperfect CSI scenario. In this case, Eq. (10) can be rewritten by considering the compensator prefilter as

$$
\boldsymbol{y}=(\hat{\boldsymbol{H}}+\boldsymbol{\Delta} \boldsymbol{H})\left(\hat{\boldsymbol{H}}_{T}+\boldsymbol{F}_{\boldsymbol{C}}\right) \boldsymbol{x}+\boldsymbol{n}
$$

The received signal at $j$-th receive antenna can be expressed by

$$
\begin{aligned}
y_{j}= & \underbrace{\sum_{i=1}^{N_{T}}\left(\hat{\boldsymbol{h}}_{i j} \overline{\boldsymbol{H}}_{i j}+\boldsymbol{\Delta} \boldsymbol{h}_{i j} \overline{\boldsymbol{F}}_{i j}\right) x_{j}}_{\text {Signal }} \\
& +\underbrace{\sum_{i=1}^{N_{T}} \sum_{\substack{k=1 \\
k \neq j}}^{N_{R}}\left(\hat{\boldsymbol{h}}_{i j} \overline{\boldsymbol{H}}_{i k}+\boldsymbol{\Delta} \boldsymbol{h}_{i j} \overline{\boldsymbol{F}}_{i k}\right) x_{k}+\sum_{i=1}^{N_{T}} \sum_{k=1}^{N_{R}}\left(\boldsymbol{\Delta} \boldsymbol{h}_{i j} \overline{\boldsymbol{H}}_{i k}+\hat{\boldsymbol{h}}_{i j} \overline{\boldsymbol{F}}_{i k}\right) x_{k}+\underbrace{n_{j}}_{\text {Noise }}}_{\text {Interference }}
\end{aligned}
$$

Then, the SINR at the $j$-th receive antenna is given by

$$
\operatorname{SINR}_{j}=\frac{\left\|\sum_{i=1}^{N_{T}} \hat{\boldsymbol{h}}_{i j} \overline{\boldsymbol{H}}_{i j}\right\|_{F}^{2}+\left\|\sum_{i=1}^{N_{T}} \boldsymbol{\Delta} \boldsymbol{h}_{i j} \overline{\boldsymbol{F}}_{i j}\right\|_{F}^{2}}{\left\|\sum_{\substack{i=1 \\ k}}^{N_{T}} \sum_{\substack{k \\ k \neq j}}^{N_{R}} \hat{\boldsymbol{h}}_{i j} \overline{\boldsymbol{H}}_{i k}\right\|_{F}^{2}+\left\|\sum_{i=1}^{N_{T}} \sum_{k=1}^{N_{R}} \hat{\boldsymbol{h}}_{i j} \overline{\boldsymbol{F}}_{i k}\right\|_{F}^{2}+d h_{j}+E\left\{\left\|n_{j}\right\|_{F}^{2}\right\}}
$$

Where the value $d h_{j}$ is defined as

$$
d h_{j}=E\left\{\left\|\sum_{i=1}^{N_{T}} \sum_{k=1}^{N_{R}} \boldsymbol{\Delta} \boldsymbol{h}_{i j} \overline{\boldsymbol{H}}_{i k}\right\|_{F}^{2}+\left\|\sum_{i=1}^{N_{T}} \sum_{\substack{k=1 \\ k \neq j}}^{N_{R}} \boldsymbol{\Delta} \boldsymbol{h}_{i j} \overline{\boldsymbol{F}}_{i k}\right\|_{F}^{2}\right\}
$$

In addition to $\boldsymbol{R}_{i j}$ and $\boldsymbol{C}_{i k}$, if we define $\boldsymbol{C}_{i k}^{\prime} \stackrel{\Delta}{=} \hat{\boldsymbol{h}}_{i j} \overline{\boldsymbol{F}}_{i k}$ as crosscorrelation vector of $\hat{\boldsymbol{h}}_{i j}$ with 
$\boldsymbol{f}_{i k}=\left[f_{i k}[0], \ldots, f_{i k}[l], \ldots, f_{i k}[L-1]\right]_{1 \times L}$ where $k \neq j$, then, the SINR at the $j^{\text {th }}$ receive antenna is obtained as

$$
\operatorname{SINR}_{j}=\frac{\sum_{i=1}^{N_{T}} \sum_{l=0}^{2 L-2}\left|\boldsymbol{R}_{i j}(l)\right|^{2}+L \sigma_{e}^{2} \sum_{i=1}^{N_{T}} \sum_{l=0}^{L-1}\left|f_{i j}(l)\right|^{2}}{\sum_{\substack{N_{T} \\ N}}^{N_{k}} \sum_{\substack{k=1 \\ k \neq j}}^{2 L-2}\left|\boldsymbol{C}_{i k}(l)\right|^{2}+\sum_{i=1}^{N_{T}} \sum_{k=1}^{N_{R}} \sum_{l=0}^{2 L-2}\left|\boldsymbol{C}_{i k}^{\prime}(l)\right|^{2}+d h_{j}+\sigma_{n}^{2}}
$$

where $d h_{j}$ is obtained as

$$
d h_{j}=L \sigma_{e}^{2} \sum_{i=1}^{N_{T}} \sum_{k=1}^{N_{R}} \sum_{l=0}^{L-1}\left|\hat{\boldsymbol{h}}_{i k}(l)\right|^{2}+L \sigma_{e}^{2} \sum_{i=1}^{N_{T}} \sum_{\substack{k=1 \\ k \neq j}}^{N_{R}} \sum_{l=0}^{L-1}\left|f_{i k}(l)\right|^{2}
$$

in which the estimation errors of the different sub-channels are assumed to be mutually independent, i.e., $\boldsymbol{\Delta} \boldsymbol{h}_{i j} \amalg \boldsymbol{\Delta} \boldsymbol{h}_{m n}$.

Power loading scheme can be obtained in the robust optimized system by replacing Eq. (27) into Eqs. (20), (21) and (22), and using the iterative procedure mentioned in [8]. If the channel estimation is assumed to be perfect, we can neglect the interference terms caused by the channel estimation error, and then the SNR can be written as

$$
S N R_{j}=\frac{\sum_{i=1}^{N_{T}} \sum_{l=0}^{2 L-2}\left|\boldsymbol{R}_{i j}(l)\right|^{2}+L \sigma_{e}^{2} \sum_{i=1}^{N_{T}} \sum_{l=0}^{L-1}\left|f_{i j}(l)\right|^{2}}{\sigma_{n}^{2}}
$$

As it can be seen from (29), $S N R_{j}$ values are larger than its counterpart in Eq. (14). Increase in the $S N R_{j}$ values in Eq.

(29) relative to Eq. (14) is because of the error compensator coefficients $f_{i j}(l)$ obtained in robust optimization scheme in

Section 3. Therefore, it is expected that the power loading scheme in robust optimization, outperforms relative to conventional TR-MIMO-UWB system in an imperfect CSI. This expression will be denoted by simulation. If $\boldsymbol{F}_{\boldsymbol{C}}=0$ then we obtain the power allocation scheme in conventional TR-MIMO-UWB system in an imperfect CSI scenario.

\section{Simulations AND RESUlts}

To evaluate the performance of the proposed optimization methods (robust optimization and power loading) for the TR-MIMO-UWB system, Monte Carlo simulations are conducted in this section. As in [5], the second-order derivative of Gaussian pulse has been used as the transmitted pulse $p(t)$, which is mathematically defined as [5]

$$
p(t)=\left[1-4 \pi\left(\frac{t-t_{c}}{T_{p}}\right)^{2}\right] e^{-2 \pi\left(\frac{t-t_{c}}{T_{p}}\right)^{2}}
$$

where $T_{p}$ is a parameter corresponding to pulse width, and $t_{c}$ is a time shifting of the pulse. In the following simulations, we consider $T_{p}=5 n s$, and $t_{c}=2.5 n s$. Also, one pulse per symbol is assumed, i.e., symbol duration $T$ is assumed $5 \mathrm{nsec}$ where is equal to transmission rate of 200Mbps with BPAM modulation and also, sampling time $t_{s}=0.167 n \mathrm{sec}$ is considered. We assume that the signal is transmitted over UWB channels and perfectly synchronized at receiver. The most widely adopted UWB multipath channel model has been proposed by the IEEE 802.15.3a Task Group [23-24]. According to this proposal, slow fading, dense multipath and quasi-statistic are the key features of the UWB channel. Four scenarios were proposed: CM1-based on line of sight (LOS) 0-4m length, CM2-based on non-LOS (NLOS) 0-4 m, CM3-based on NLOS 4-10 m, and CM4 based on an extreme NLOS environment. As in [5], we use the IEEE 802.15.3a CM4 channel model for each channel in simulations to evaluate our proposed solutions in the worst case scenario of the indoor multipath channels.

To optimize the TR-MIMO-UWB system performance of Fig. 1 and to compensate the channel estimation error by prefilter, we use the robust optimization scenario mentioned in Section 3. The entries of $\boldsymbol{\Delta H}$ are assumed to be zero mean i.i.d. complex Gaussian random variables. In our simulations, we consider 3 values for the estimation error

variance as $\sigma_{e}^{2}=0.1,0.2$ and 0.3 . The performance of uncoded TR-MIMO-UWB system with robust optimization scheme is shown in Fig. 2 in imperfect CSI. It can be seen that, for the particular values of the estimation error variance considered in this section, the BER performance of $2 \times 2$ TR-MIMO-UWB is considerably improved. For example, with $\sigma_{e}^{2}=0.1$, the SNR can be improved about $4 \mathrm{~dB}$ at the average $\mathrm{BER}=10^{-3}$.

In Figs. 3 and 4, the equivalent CIRs for a $2 \times 2$ TR-MIMOUWB system are compared in perfect and imperfect CSI where $\sigma_{e}^{2}=0.1$ is assumed. It should be noted that the signal transmitted over the desired channel is focused into a narrow time instant, while ISI channel spreads the signal. This property is useful for ISI mitigation when the signal is transmitted over long delay spread channel [5]. But according to Figs. 3 and 4, in an imperfect CSI, the delay spread of the equivalent channel is increased as compared with perfect CSI. This channel imperfection degrades the performance of TR-MIMO-UWB system. As it is shown in Figs. 3 and 4, the time focusing feature of the TR technique in the robust optimized TR-MIMO-UWB system is yet preserved. Figs. 3 and 4 denote the temporal focusing property of TR technique in four sub-channels, before and after the robust optimization. Someone can understand the time compression of the energy (power) at the center of the compressed equivalent TR response from these figures. But, it might be possible for another one to feel and understand the time focusing of TR method by focusing gain better than the plotted figures. For more understanding of this, according to [25]-[26], focusing gain (FG) is defined as the ratio of the strongest tap power to the total tap power of the received equivalent $\mathrm{TR}$ response. If we define the equivalent TR response between $i$-th transmit antenna and $j$-th receive antenna as $\overline{\boldsymbol{h}}_{i j}=\left[\bar{h}_{i j}[-(L-1)], \ldots, \bar{h}_{i j}[0], \ldots, \bar{h}_{i j}[(L-1)]\right]$, then focusing gain ( $F G_{i j}$ ) according to [25]-[26] is given by

$$
F G_{i j}=\frac{\left|\bar{h}_{i j}[0]\right|^{2}}{\sum_{l=-(L-1)}^{L-1}\left|\bar{h}_{i j}[l]\right|^{2}}
$$

We obtained this ratio by averaging over 100 multipath channel realizations between transmit and receive antennas and tabulated in Table 1. As it can be seen from Table 1, the FG in robust optimized TR scheme is more than the imperfect CSI case and less than the perfect CSI scenario. This result proves the performance improvement of Fig. 2 and the temporal focusing of Figs. 3 and 4.

In addition to the robust optimization scheme, we use the 
proposed power loading solution mentioned in Section 4 to improve the TR-MIMO-UWB system performance more than ever. In Fig. 5, we can observe the performance of the proposed power loading for the robust optimization. As can be seen from this figure, for instance in $\sigma_{e}^{2}=0.1$, the performance of power loading is noticeable, especially for high SNR values. Also, as it can be seen from Table 1, the FG in the power loading scheme is more than the robust optimization scheme. This means that the power loading method outperforms the robust optimization scheme. For validity of this, as it can be realized from Fig. 5, the power loading scheme has better performance relative to robust optimization method, especially for high SNR. Such as, according to Fig. 5, for $\sigma_{e}^{2}=0.1$, the power loading scheme substantially outperforms the robust optimization method about $2 \mathrm{~dB}$ SNR at the average $\mathrm{BER}=10^{-3}$.

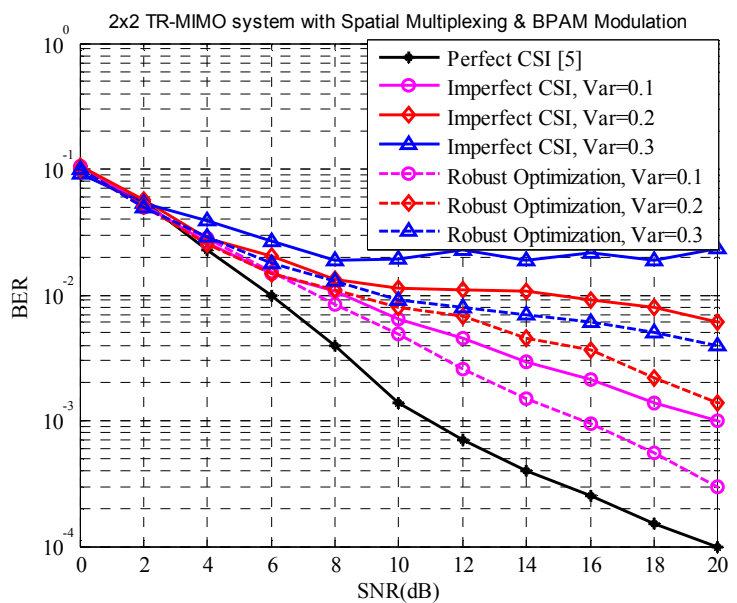

Fig. 2. BER performance of $2 \times 2$ TR-MIMO-UWB with robust optimization.
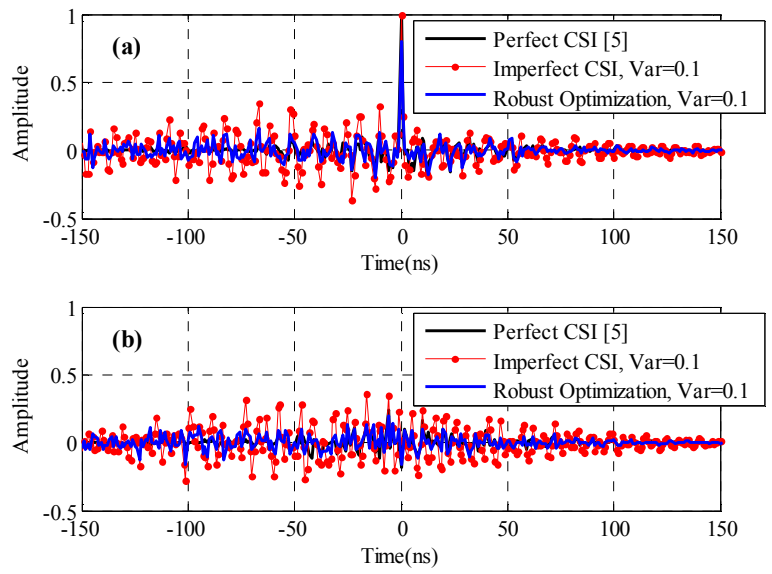

Fig. 3. Plotting (a) $\overline{\boldsymbol{h}}_{11}$ and (b) $\overline{\boldsymbol{h}}_{21}$ of the matrix $\boldsymbol{H}_{T R}$ (Impulse response of $2 \times 2$ TR-MIMO-UWB equivalent channel with pulse shaping).
TABLE I

COMPARISON OF THE PROPOSED SCHEMES (ROBUST OPTIMIZATION \& POWER LOADING) IN FOCUSING GAIN TERM BEFORE AND AFTER OPTIMIZATION FOR $\sigma_{e}^{2}=0.1$

\begin{tabular}{ccccc}
\hline \hline $\begin{array}{c}\text { Sub-channel } \\
\text { Index }\end{array}$ & $\begin{array}{c}\text { Perfect } \\
\text { CSI }\end{array}$ & $\begin{array}{c}\text { Imperfect } \\
\text { CSI }\end{array}$ & $\begin{array}{c}\text { Robust } \\
\text { Optimization } \\
\text { Scheme }\end{array}$ & $\begin{array}{c}\text { Power Loading } \\
\text { Scheme }\end{array}$ \\
\hline$\overline{\boldsymbol{h}}_{11}$ & 0.6621 & 0.2264 & 0.3740 & 0.5643 \\
$\overline{\boldsymbol{h}}_{21}$ & 0.0792 & 0.0236 & 0.0375 & 0.0534 \\
$\overline{\boldsymbol{h}}_{12}$ & 0.0769 & 0.0214 & 0.0332 & 0.0521 \\
$\overline{\boldsymbol{h}}_{22}$ & 0.6577 & 0.2163 & 0.3565 & 0.5536 \\
\hline \hline
\end{tabular}
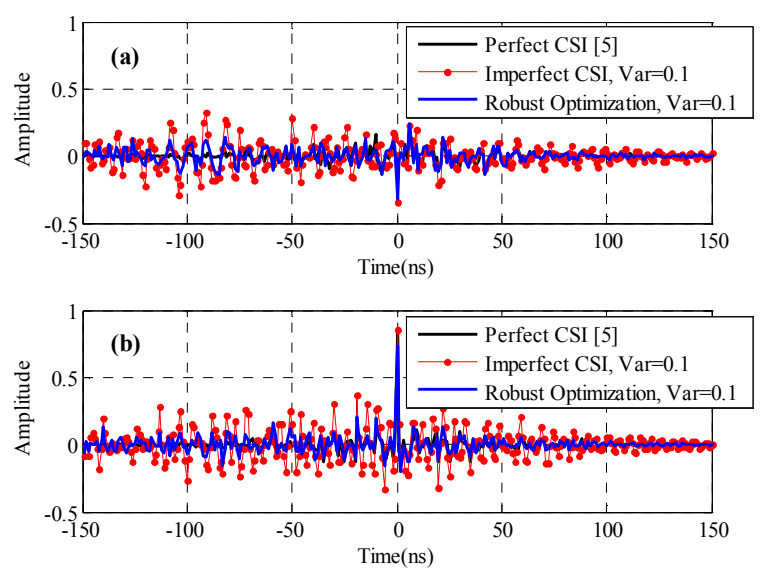

Fig. 4. Plotting (a) $\overline{\boldsymbol{h}}_{12}$ and (b) $\overline{\boldsymbol{h}}_{22}$ of the matrix $\boldsymbol{H}_{T R}$ (Impulse response of $2 \times 2$ TR-MIMO-UWB equivalent channel with pulse shaping).

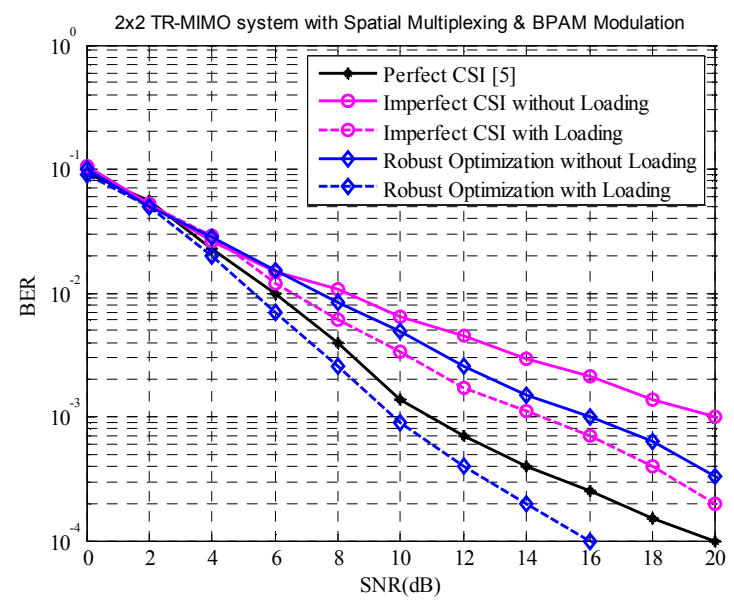

Fig. 5. Power loading in robust optimization for imperfect CSI and $\sigma_{e}^{2}=0.1$.

\section{CONCLUSION}

The imperfect CSI effects, caused by channel estimator error, on TR-MIMO-UWB system were considered in this paper. At first, a pre-filtering approach as robust optimization scheme based on MMSE equalizer is developed. We observed that the robust optimization technique considerably improved the BER performance of $2 \times 2$ TR-MIMO-UWB system, where temporal focusing feature of the TR technique in the robust optimized TRMIMO-UWB system was kept, especially for high SNR values. For instance, $2 \times 2$ TR-MIMO-UWB system with the 
proposed robust optimization scheme was improved about $4 \mathrm{~dB}$ SNR at the average $\mathrm{BER}=10^{-3}$ for $\sigma_{e}^{2}=0.1$. Then, the power loading strategy was proposed for the imperfect CSI and the robust optimized cases. It was observed that it brought a performance gain in the $2 \times 2$ TR-MIMO-UWB system in an imperfect CSI scenario and the robust optimized system. Also, it was seen that the power loading scheme has better performance relative to robust optimization method, especially for high SNR. For example in $\sigma_{e}^{2}=0.1$, the power loading scheme substantially outperforms the robust optimization method about $2 \mathrm{~dB}$ SNR at the average $\mathrm{BER}=10^{-3}$.

Note that we proposed the general new solutions for the wireless personal area network (WPAN) TR-MIMO-UWB systems in this paper. It is clear that our methods can be especially used in the extremely multipath indoor environments as the short-range high-data rate transmission, such as, downlink scenario of the access point to the handsets in the indoor environments, the multimedia and other high-data rate transmissions in a wireless home networking and so on.

\section{REFERENCES}

[1] F. Nekoogar, "Ultra-wideband communications: fundamentals and applications", Pearson Education, First printing, September 2005.

[2] Jeffrey H. Reed, "An Introduction to Ultra Wideband Communication Systems", Prentice Hall PTR, April 05, 2005.

[3] G. Foschini and M. Gans, "on the limits of wireless communications in a fading environment when using multiple antennas", Wireless Pers. Commun, 1998, 6, (3), pp.311-335.

[4] C. Zhou, N. Guo and R. C. Qiu, "Time-Reversed Ultra wideband (UWB) Multiple Input-Multiple Output (MIMO) Based on Measured Spatial Channels", IEEE Transactions on Vehicular Technology, 2009 , p.p. $1-15$.

[5] H. Nguyen, F. Zheng and T. Kaiser, "Antenna selection for time reversal MIMO UWB systems", In IEEE 69th Vehicular Technology Conf., Barcelona, Spain, 26-29 April 2009.

[6] T. Kaiser and F. Zheng, "Ultra Wideband Systems with MIMO", John Wiley \& Sons Ltd.,UK, 2010.

[7] R. J. Cramer, R. A. Scholtz, and M. Z. Win, "Evaluation of an UltraWide-Band Propagation Channel," IEEE Trans. Ant. Prop., Vol. 50, No. 5, pp. 561-570, May 2002.

[8] R. J. Cramer, "An evaluation of ultra wideband propagation channels," Ph.D. dissertation, Univ. Southern Calif., Los Angeles, CA, Dec. 2000.

[9] T. Strohmer, M. Emami, J. Hansen, G. Papanicolaou, and A. J. Paulraj, "Application of time reversal with MMSE equalizer to UWB Communications", Proc. IEEE Global Telecommunications Conference, Dallas, Texas, Nov.29-Dec.3, 2004, pp.3123-3127.

[10] P. Kyristi, G. Panicolaou, and A. Oprea, "MISO time reversal and 918 delay-spread compression for FWA channels at $5 \mathrm{GHz}$," IEEE Antennas 919 Wireless Propag. Lett., Dec. 2004, vol. 3, pp. 96-99.

[11] I. H. Naqvi, P. Besnier and G. El Zein, "Robustness of a time-reversal ultra wideband system in non-stationary channel environment", IET Microwaves, Antennas and Propagation, March 2011, Vol. 5, No. 4, pp. 468-475.

[12] X. Liu, B. Z. Wang, S. Xiao and S. Lai, "Post-Time-Reversad MIMO Ultrawideband Transmission Scheme", IEEE Transactions on Antennas and Propagation, May 2010, vol. 58, no.5, pp.1731-1738.

[13] H. Khaleqhi Bizaki, S. Alizadeh, "Mitigation of Channel Estimation Error in TR-UWB system based on a Novel MMSE Equalizer", Springer Annals of Telecommunications, doi: 10.1007/s12243-0120325-8, published online: 06 Oct. 2012.

[14] S. Alizadeh, H. Khaleqhi Bizaki and M. Okhovvat, "Effect of Channel Estimation Error on Performance of Time Reversal-UWB Communication System and its Compensation by Pre-filter", IET Communications, vol. 6, no. 12, pp.1781-1794, Nov. 2012.

[15] H. K. Bizaki and A. Falahati, "Tomlinson-Harashima precoding with imperfect channel state information", IET. Communication journal, vol.2, no.1, pp.151-158, January 2008.

[16] C. Windpassinger, "Detection and Pre-coding for Multiple Input Multiple Output Channels," Ph.D. dissertation, University of Erlangen, Nürnberg, June 2004.

[17] K. B. Petersen, and M. S. Pedersen, "The Matrix Cookbook", Feb. 2006, page 17

[18] C. D. Manning, P. Raghavan and H. Schütze, "An Introduction to Information Retrieval”, Cambridge University Press, Cambridge, England, Chapter 18, April 2009.

[19] K. Witrisal, "Statistical analysis of the IEEE 802.15.4a UWB PHY over Multipath Channels", IEEE Wireless Communications and Networking Conference, WCNC2008, March 31-April 32008.

[20] D. Abbasi-moghadam, V. Tabataba Vakili, "Characterization of Indoor Time Reversal UWB Communication Systems: Spatial, Temporal and Frequency Properties", Wiley International Journal of Communication Systems, doi:10.1002/dac.1140, published online 28 April 2010.

[21] R.M. Corless, G.H. Gonnet, D.E.G. Hare, D.J.Jeffrey, and D.E. Knuth, "On the Lambert W Function", Advances in Computational Mathematics, volume 5, 1996, pp. 329-359.

[22] T. Hunziker, D. Dahlhaus, "Optimal Power Adaptation for OFDM Systems with Ideal Bit-Interleaving and Hard-Decision Decoding", IEEE International Conference on Communications (ICC), vol. 5, 2003, pp:3392-3397.

[23] J. Foerster et al., "Channel modeling sub-committee report final," IEEE P802.15 Wireless Personal Area Networks, P802.15-02/490r1$S G 3 a$, Feb. 2003.

[24] A. F. Molisch, K. Balakrishnan, D. Cassioli, C. Chong, S. Emami, A. Fort, J. Karedal, J. Kunisch, H. Schantz, U. Schuster, and K. Siwiak, "IEEE 802.15.4a Channel model final report," Tech. Rep.

[25] A. Akogun, R. C. Qiu, and N. Guo, "Demonstrating time-reversal in ultra-wideband communications using time domain measurements", The Instrumentation, Systems and Automation (ISA) 51st International Instrumentation Symposium, Knoxville, Tennessee, May 2005, pp.1-5.

[26] A. E. Akogun, "Theory and application of time reversal technique to ultra-wideband wireless communication", MSc. dissertation, Tennessee Technological University, August 2005.

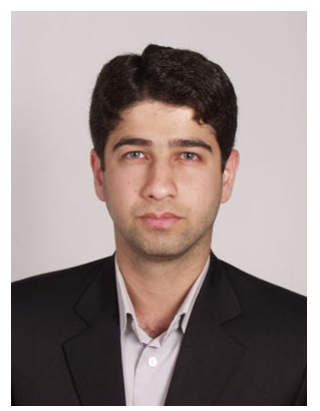

Sajjad Alizadeh was born in Roudsar, Guilan Province, Iran on November 3, 1983. He received the Associate Degree (A.D.) from Electronics Engineering College (Electronics Industry University) of Shiraz University (Shiraz University of Technology (SUTECH) today), Shiraz, Iran, in 2005 and the B.Sc. degree from Shahid Rajaee Teacher Training University (SRTTU), Tehran, Iran, in 2007, both in electronics. Also he received the M.Sc. degree in Communication Engineering from Imam Hossein University (IHU), Tehran, Iran, in 2011. He has served as a referee in IET Communications journal. His current research interests are in the area of UWB wireless communications with special emphasis on digital and statistical signal processing, Time Reversal signaling, propagation channels modeling, multiple-antenna (MIMO) schemes, Cognitive Radio networks, and blind detection and parameters estimations in spread spectrum systems.

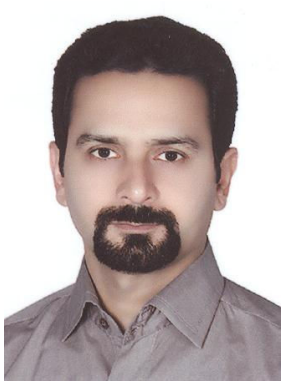

Signal Processing.
Hossein Khaleghi Bizaki received his $\mathrm{PhD}$ degree in Electrical Engineering, Communication system, from Iran University of Science and Technology (IUST), Tehran, Iran, in 2008. Dr. Bizaki started his work as assistant professor in 2008. Now, he is author or coauthor of more than 30 publications. His research interests include Information Theory, Coding Theory, Wireless Communication, MIMO Systems, Space Time Processing, and other topics on Communication System and 